ESJ Humanities

\title{
Contribution des Techniques Andragogiques au Succês de la Formation Continue : Cas de la Mise en Euvre des Objectifs de Développement Durable au Benin
}

Florentine Akouété-Hounsinou

Centre béninois de Recherche scientifique et de l'Innovation (CBRSI)

Doi:10.19044/esj.2021.v17n42p23

Submitted: 17 November 2021

Accepted: 02 December 2021

Published: 31 December 2021

\author{
Copyright 2021 Author(s) \\ Under Creative Commons BY-NC-ND \\ 4.0 OPEN ACCESS
}

Cite As:

Akouété-Hounsinou F. (2021). Contribution des Techniques Andragogiques au Succês de la Formation Continue : Cas de la Mise en Euvre des Objectifs de Développement Durable au Benin. European Scientific Journal, ESJ, 17 (42), 23.

https://doi.org/10.19044/esj.2021.v17n42p23

\section{Résumé}

Dans cet article, le travail a consisté à mesurer l'atteinte des objectifs de la formation et à analyser la perception que les participants ont de leurs acquis de formation. Ainsi, cette recherche a pour but d'apprécier le recours aux techniques andragogiques dans la formation des formateurs d'adultes dans un centre de formation professionnelle continue. La démarche méthodologique adoptée est mixte. Le recensement exhaustif des 12 formateurs en Objectifs de Développement durable (ODD) conviés au renforcement de leurs capacités est fait. Il y en a eu 10 qui ont accepté de participer à l'enquête. La collecte des données a été réalisée grâce à un questionnaire (fiche d'évaluation de la formation) ayant servi à voir leur niveau de satisfaction et un guide d'entretien pour connaître la perception des participants sur l'atelier de formation. L'analyse catégorielle a permis de traiter les données qualitatives et de répartir les différentes réponses dans des ensembles en fonction de leur signification et de leur interprétation contextuelle. Majoritairement, les participants formateurs sont totalement satisfaits des modules de formation en andragogie qui, avec les notions enseignées en ODD, permettront l'amélioration de leurs pratiques. Plus de $70 \%$ des participants ont aimé l'organisation matérielle et plus de $80 \%$ ont nettement apprécié le déroulement de la formation. Il importe donc de former les formateurs à l'approche andragogique pour renforcer leurs capacités dans la compréhension et l'interprétation des ODD comme stratégie pour l'atteinte 
des objectifs de formation.

Mots-clés : Adultes, andragogie, formation, apprentissage, Objectifs de Développement durable

\title{
Contribution of Andragogic Techniques to the Success of Continuing Education : Case of the Implementation of the Sustainable Development Goals in Benin
}

\author{
Florentine Akouété-Hounsinou \\ Centre béninois de Recherche scientifique et de l'Innovation (CBRSI)
}

\begin{abstract}
In this article, the work consisted of measuring the achievement of training objectives and analyzing participants' perception of their training outcomes. Thus, the purpose of this research is to assess the use of andragogic techniques in the training of adult trainers in a continuing vocational training centre. The methodological approach adopted is mixed. The exhaustive census of the 12 trainers in the Sustainable Development Goals (SDGs) invited to strengthen their capacities is done. There were 10 who agreed to participate in the survey. Data collection was carried out through a questionnaire (training score sheet) used to see their level of satisfaction and an interview guide to find out how participants perceived the training workshop. Categorical analysis made it possible to process qualitative data and to divide the different responses into sets according to their meaning and contextual interpretation. For the most part, the training participants are completely satisfied with the training modules in andragogy which, together with the concepts taught in the SDGs, will allow the improvement of their practices. More than $70 \%$ of the participants liked the material organization and more than $80 \%$ clearly appreciated the course of the training. It is therefore important to train trainers in the andragogic approach to strengthen their capacities in understanding and interpreting the SDGs as a strategy for achieving training objectives.
\end{abstract}

Keywords: Adults, andragogy, training, learning, Sustainable Development Goals

\section{Introduction}

L'andragogie fait partie des Sciences de l'Éducation. Mais, le concept est beaucoup moins usité que celui de pédagogie et du qualificatif pédagogique qui se retrouve aisément dans les discours sur l'andragogie. Cette 
dernière comporte des particularités qu'il importe de considérer. Health Nexus santé (2003, p. 1) a expliqué qu'

En andragogie, la situation d'apprentissage : favorise un climat d'apprentissage informel, détendu, égalitaire, convivial, centré sur l'estime de soi, le désir de collaboration et les besoins des apprenants ; permet la référence de l'adulte à ses expériences qui constituent une ressource riche et fait appel à son autonomie, sa capacité d'adaptation au changement, sa motivation intrinsèque. Le facilitateur favorise l'exploitation de ces ressources et maintient un équilibre entre la structure de formation et le degré d'autonomie laissé à l'apprenant.

Ce passage donne une idée globale de ce que l'andragogie, par essence, recherche. Les sensibilités, connaissances et compétences issues du vécu de l'adulte font qu'il n'est pas comme l'enfant ou l'adolescent qui ne demande qu'à être orienté. En fait, a souligné Akouété-Hounsinou (2021), les modules de formation et les activités qui sont données (à des adultes) sont appelés à soutenir l'apprentissage pour que les apprenants soient satisfaits. Selon Bourgeois et Nizet (1997), Mezirow (1991), Elias et Merriam (1983) et Cross (1981) cités par Health Nexus santé (2003), l'adulte réussit à étudier grâce à de la motivation et de la détermination, et lorsqu'il est respecté en tant qu'individu, mais également en tant qu'apprenant. Dans un tel contexte, les prérequis sont valorisés; ce qui permet l'amélioration des compétences antérieures au sein du milieu d'apprentissage et de formation. Pour garantir ces apprentissages, l'adulte a besoin comme tout apprenant d'être dans un environnement physique propice. Nelson (2020) a suggéré de comprendre par environnement physique d'apprentissage, les structures physiques de l'environnement scolaire c'est-à-dire l'ensemble des espaces, de l'équipement et des outils de l'école et, par extension, du milieu de formation.

$\mathrm{Au}$ Bénin, comme peut-être dans d'autres pays, l'Approche Par Compétences (APC) et le système Licence-Master-Doctorat permettent d'asseoir un dispositif qui tienne mieux compte de l'apprenant. Dans un tel contexte, ces dernières années ont vu se renforcer l'intérêt pour la satisfaction de l'adulte apprenant en formation. Pour l'expliquer, la plupart des auteurs consultés (Health Nexus santé, 2003 ; Knowles, 1967 ; Expilly, 2017) ont mis l'accent sur les étapes du processus de gestion de la formation. Il faut retenir :

- l'analyse des besoins de formation identifiés ;

- la formulation des objectifs d'apprentissage ;

- l'organisation des sessions de formation (conception du contenu et planification);

- le déroulement de la formation ;

- l'évaluation des acquis de la formation sur le site de la formation et dans le milieu professionnel d'origine. 
Issus des besoins de formation, les objectifs d'apprentissage, dès qu'ils sont définis correctement, permettent de poursuivre aisément les autres phases du processus. Dès que de nouveaux besoins apparaissent, le cycle reprend. Zaoré (2007) a précisé que la formation des adultes est différente de celle du système éducatif classique, car elle vise un perfectionnement et un élargissement des compétences permettant aux apprenants de s'adapter davantage aux changements qui surgissent dans leur milieu de travail. Pour rendre la formation optimale, Knowles (1990) a recommandé :

de tenir compte de l'environnement (physique et relationnel) pour instaurer un climat propice à l'apprentissage, de diagnostiquer les besoins d'apprentissage, de créer un mécanisme de planification et de décision incluant les apprenants, de formuler les objectifs du programme, de concevoir des expériences de l'apprentissage, de penser l'évaluation de la formation (par le formateur et les apprenants). (Cieuta, 2019, p. 1)

En effet, comme l'a exprimé Health Nexus santé (2003), «le développement d'une stratégie de formation en andragogie exige une approche systémique ». Schwartz (1968) a parlé plutôt d'éducation pour adulte et en a distingué trois types: l'éducation en milieu socio-culturel, l'éducation en milieu socio-professionnel et l'éducation post-universitaire. La première forme est décrite comme celle des mouvements d'éducation citoyenne. La deuxième entend répondre à des problèmes particuliers qui naissent dans le milieu socio-professionnel. La troisième envisage de mettre à jour les connaissances et compétences.

Par ailleurs, Health Nexus santé (2003) a recommandé qu'

Un programme de formation efficace considère les changements de comportement comme un processus de l'apprentissage. La formation est le moyen le plus direct d'améliorer la performance, en particulier si elle porte non seulement sur l'acquisition de connaissances mais aussi sur les attitudes des apprenants et les compétences requises pour agir. En tant que responsable de la formation, vous devez veiller à ce que vos formations soient appliquées de manière stratégique en vue de renforcer l'impact de vos programmes sur la vie de vos bénéficiaires.

Selon Yennek (2015), la formation focalisée sur l'apprenant adulte doit être associée à son milieu professionnel et suscité un intérêt personnel quelconque. C'est dire que lorsque la formation des adultes se fait selon les normes andragogiques, les participants sont plus réceptifs à l'enseignement et se prêtent facilement aux activités qui leur sont proposées.

Meilleur (2018) a fait le point de l'essentiel des travaux de Knowles (1970-1990) qui ont posé les postulats qui soutiennent la réflexion et les 
pratiques andragogiques : le concept de soi chez l'adulte ; l'expérience de l'apprenant adulte ; la volonté d'apprendre ; l'orientation de l'apprentissage ; et enfin la motivation à apprendre. Les fondamentaux de la formation des adultes, qui en découlent, peuvent se formuler ainsi qu'il suit :

- l'implication de l'adulte dans le processus de formation est primordiale pour permettre les apprentissages ;

- l'expérience et le droit à l'erreur sont à prendre en compte car induisent de meilleures réactions lors des activités d'apprentissage ;

- la vie professionnelle et/ou personnelle de l'adulte inspire les activités proposées de sorte que les connaissances et les compétences acquises servent dans celle-ci à court terme ;

- la résolution de problèmes et des activités pratiques favorisent l'apprentissage.

Il convient donc de relever qu'en andragogie, le formateur, acteur par excellence du processus, intervient aussi comme un réalisateur. Autrement, après avoir fait tout le préalable et conçu les modules de formation, le formateur va coordonner chaque activité pour amener les apprenants vers les apprentissages qu'il désire. Pour Expilly (2017), le formateur est celui qui fait se développer les compétences, c'est-à-dire qui travaille non seulement sur le savoir, mais aussi sur le savoir-faire et le savoir-être de ses apprenants. Health Nexus santé (2003) a précisé que « Le rôle d'un formateur est de répondre aux besoins identifiés pour un changement de comportement et une performance spécifique ». Suivant les principes fondamentaux et les pratiques usuelles en matière d'andragogie à partir des données de la littérature, les principes fondamentaux sont opérationnalisés de la façon suivante :

- prise en compte de l'expérience des apprenants ;

- responsabilisation des apprenants pendant la formation ;

- adéquation de la formation avec l'intérêt des apprenants pour mieux favoriser l'apprentissage ;

- forte implication des apprenants dans le déroulement des activités de formation.

Zaoré (2007) a rappelé que le formateur doit concevoir des feedbacks adaptés pour maintenir l'estime de soi : l'adulte n'apprend qu'en mettant en pratique l'enseignement reçu pour se l'approprier. Pour Schmidt (2018),

Le formateur aura, dès lors, plus un rôle de facilitateur, de médiateur entre le contenu et l'adulte apprenant, mais aussi sur la relation entre les participants. Il encourage, il stimule, il relance l' apprenant qui apprend à s'auto-évaluer. L'être humain est très complexe, nous sommes cependant probablement tous d'accord pour dire qu'il 
possède une grande capacité de réflexion et de créativité. L'application des principes d'andragogie encourage ce processus de réflexion et de créativité ; stimulant ainsi chez l'apprenant son besoin intrinsèque de se dépasser et par conséquent établit les fondements du changement de comportement et l'adoption de nouvelles attitudes. (p.6)

En réalité, la démarche andragogique repose non seulement sur la détermination des nouveaux savoir-faire et savoir-être à opérer, mais aussi sur le processus d'acquisition des connaissances et des compétences.

Le projet d'article s'est vu porter par une session de formation organisée en juillet 2021 dans un centre de formation continue de l'Ecole nationale d'Administration et de Magistrature (ENAM) du Bénin. Selon l'approche de ce centre, toute session de formation continue des formateurs comprend la mise à niveau des compétences de base des formateurs en andragogie (formation des adultes). Cette session de formation s'est penchée sur la mise en œuvre des Objectifs de Développement durable. L'article met en exergue les principes et les outils de formation en andragogie et la perception de celle-ci par les personnes adultes ayant participé à ladite formation. Ce travail a donc pour but de faire connaître l'importance de la formation des formateurs aux techniques andragogiques dans l'atteinte des objectifs de formation initiale ou continue. Plus spécifiquement, il s'agit :

- de mesurer l'atteinte des objectifs de la formation dans le respect des principes andragogiques ;

- d'analyser la perception que les participants ont de leurs acquis après la formation.

\section{Méthodes}

Le cadre de la recherche est l'Université d'Abomey-Calavi (UAC) qui est une université publique située dans la commune d'Abomey-Calavi, proche de celle de Cotonou, capitale économique du Bénin.

La démarche méthodologique adoptée est quantitative et qualitative. La population cible est composée d'enseignants-chercheurs de l'Ecole nationale d'Administration du Bénin (ENA-Bénin) et d'experts formateurs en Objectifs de Développement durable (ODD), qui sont des cadres supérieurs de l'administration publique béninoise. La session de formation a été organisée au niveau national. L'enquête a consisté à déployer un guide d'entretien semidirectif auprès des participants formateurs en ODD et à évaluer la formation par le remplissage de fiches (questionnaire). Les outils de collecte des données ont été préalablement testés avant l'enquête proprement dite. Tous les participants sont pris en compte, car un recensement exhaustif de l'ensemble des participants a été réalisé. Ce sont 12 formateurs (trois enseignants- 
chercheurs de l'ENA-Bénin et neuf experts en ODD) qui ont suivi la formation ; 10 parmi eux ont accepté de participer à l'enquête.

L'analyse des données qualitatives a été réalisée avec le logiciel Modalisa qui permet, à lui seul, le traitement de toutes les données d'enquête. Il s'agit essentiellement de l'évaluation du niveau de satisfaction des participants à l'atelier de formation des formateurs sur la base de l'approche andragogique. Un rapprochement est fait entre le niveau de satisfaction exprimé par les participants et l'adéquation des principes avec les outils andragogiques mobilisés et mis en place pendant la formation.

En tenant compte des particularités de la formation des adultes, le questionnaire comporte des critères soumis à une échelle d'appréciation (de Likert, 1961) à quatre niveaux. Ces niveaux sont conceptualisés comme suit : Tout à fait satisfait(e) ou 4 ; En partie satisfait(e) ou 3 ; Insuffisamment satisfait(e) ou 2; Pas du tout satisfait(e) ou 1. Ce qui est à retenir du dépouillement de leurs réponses est organisé ci-dessous en guise de résultats de l'efficacité de l'approche andragogique adoptée lors de cette session de formation.

L'analyse catégorielle a permis de traiter les données qualitatives et de ranger les différentes réponses dans des ensembles en fonction de leur signification et de leur interprétation contextuelle.

Il y a lieu de signaler que l'étude gagnerait en précision si les bénéficiaires de la formation ainsi évalués faisaient l'objet d'une double évaluation (la première évaluation étant celle objet du présent article), la seconde étant destinée à apprécier le transfert de leurs acquis de la formation en milieu professionnel (c'est-à-dire pendant qu'ils animent une formation continue des adultes), plus précisément pendant la formation des cadres béninois sur la mise en œuvre des Objectifs de Développement durable.

\section{Résultats}

Les résultats sont organisés en quatre points. Chacun d'eux met en relation l'appréciation de la situation et/ou l'opinion des participants avec le contenu de la formation, les principes appliqués pendant la formation ou les outils et les techniques/méthodes andragogiques utilisés lors de la formation.

\section{De l'atteinte des objectifs de formation en matière d'andragogie appliquée aux ODD}

Le tableau 1 décrit le niveau de satisfaction exprimé par les participants pour les modules sur l'andragogie. 
Tableau 1. Le niveau de satisfaction des participants par rapport au déroulement des différents modules de formation en andragogie

\begin{tabular}{|c|c|c|c|c|}
\hline $\begin{array}{c}\text { Niveau de satisfaction par rapport } \\
\text { différents modules de la formation } \\
\text { réalisés }\end{array}$ & $\begin{array}{c}\text { Tout à } \\
\text { satisfait(e) }\end{array}$ & $\begin{array}{c}\text { En partie } \\
\text { satisfait(e) }\end{array}$ & $\begin{array}{c}\text { Insuffisamment } \\
\text { satisfait(e) }\end{array}$ & $\begin{array}{c}\text { Pas du tout } \\
\text { satisfait(e) }\end{array}$ \\
\hline Module 1 : Concepts de formation & $100 \%$ & $0 \%$ & - & - \\
\hline $\begin{array}{c}\text { Module 2 : Principes fondamentaux de } \\
\text { l'Andragogie et le rôle du formateur }\end{array}$ & $100 \%$ & $0 \%$ & - & - \\
\hline $\begin{array}{c}\text { Module 3: Objectifs d'apprentissage, } \\
\text { conception et développement des actions de } \\
\text { formation sur les ODD }\end{array}$ & $90 \%$ & $10 \%$ & - & - \\
\hline $\begin{array}{c}\text { Module 4 : Méthodes et techniques de } \\
\text { formation des adultes sur les ODD : vue } \\
\text { d'ensemble }\end{array}$ & $90 \%$ & $10 \%$ & - & - \\
\hline
\end{tabular}

Source : Fiches d'évaluation de la formation des formateurs sur l'andragogie appliquée aux stratégies de mise en ouvre des ODD.

Le tableau 1 indique qu'au niveau de chacun des quatre modules thématiques déroulés, le niveau de satisfaction des participants est très élevé. Le module portant sur les concepts de formation et celui sur des principes fondamentaux de l'andragogie et du rôle du formateur, ont apporté une pleine satisfaction aux participants. Les modules sur la formulation des objectifs d'apprentissage sur les ODD et sur les méthodes et techniques de formation des adultes sur les ODD ont obtenu un taux de satisfaction de $90 \%$ chacun. Les différentes phases de déroulement de la formation se trouvent décrites dans l'encadré 1 :

Encadré 1 : Point de la formation par les apprenants

Pour les cinq jours de formation, le chronogramme présenté dans le cahier du participant a permis d'avoir un aperçu général de la gestion du temps et des connaissances/pratiques à expérimenter durant cette session, sachant que le programme d'une journée-type de formation permet d'abord de faire l'évaluation de la veille à partir d'une fiche disponible à cet effet, et ensuite, de dérouler les activités du jour. Pendant les cinq jours de formation, les thématiques développées ont porté sur :

- Les concepts de formation et l'ingénierie de formation ;

- Les théories et modèles pédagogiques ;

- Les principes fondamentaux de l'andragogie et le rôle du formateur ;

- La formulation des objectifs d'apprentissage ;

- Les méthodes/techniques d'enseignement/d'apprentissage selon les normes andragogiques ;

- Les méthodes et outils d'évaluation des actions de formation des adultes.

Ces différentes thématiques sont introduites par la présentation d'un bref exposé de la formatrice ponctuée des interventions des participants. La 
projection de séquences de vidéo a tout aussi permis de renforcer les notions développées et les pratiques andragogiques exposées. A la suite de ces présentations, des travaux d'abord individuels et ensuite de groupes inspirés du contenu des modules de formation sur les ODD, précèdent leur mise en commun en plénière aux fins d'amendements / de validation de tous les participants. Les travaux en plénière ont été de véritables occasions d'échanges très riches en apprentissage de savoirs, de savoir-faire et de savoir-être.

\subsection{Des acquis de la formation en andragogie}

Après le déroulement de la formation, les participants se sont prononcés sur les notions apprises. L'essentiel est consigné dans l'encadré 2 :

Encadré 2 : Perception des apprenants de la formation complète déroulée Il importe pour nous de mettre l'accent sur la plus-value de la formation. On peut notamment retenir :

- le renforcement des acquis et la pertinence du contenu de formation ;

- la bonne formulation des objectifs d'apprentissage axés sur ;

- la structuration du cours sur les ODD ponctuée d'exercices pratiques ;

- les approches de solution visant à lever les obstacles d'apprentissage ;

- l'appropriation par les participants des modalités de mise en œuvre des différentes méthodes / techniques clés de formation des adultes telles que : le jeu de rôle, la simulation, les études de cas, la démonstration ;

- le caractère complémentaire des théories/modèles d'apprentissage ;

- la nécessité pour le formateur d'adapter la méthode/technique de formation en fonction des objectifs d'apprentissage préformulés et de sa cible ;

- la nécessité de conduire les évaluations formatives tout au long des séquences d'apprentissages ;

- la conduite et le suivi de l'évaluation ;

$\circ$ une meilleure préparation des sessions de formation.

\section{De la satisfaction des apprenants quant au dispositif mis en ouvre pendant la session de formation}

Le tableau 2 présente les résultats du dépouillement du questionnaire administré à la fin de la session de formation. Cette présentation concerne le dispositif mise en œuvre pendant la formation. Les résultats indiquent de manière globale que, pendant la session de formation, le dispositif mis en 
œuvre a bien fonctionné et est largement apprécié de la dizaine de participants ayant rempli le questionnaire.

Tableau 2. Le niveau de satisfaction des participants par rapport aux capacités en matière d'andragogie et de prise en compte des ODD dans les pratiques

\begin{tabular}{|c|c|c|c|c|}
\hline $\begin{array}{c}\text { Capacités en matière d'andragogie et de prise } \\
\text { en compte des ODD dans les pratiques }\end{array}$ & $\begin{array}{c}\text { Tout à } \\
\text { satisfait(e) }\end{array}$ & $\begin{array}{c}\text { En partie } \\
\text { satisfait(e) }\end{array}$ & $\begin{array}{c}\text { Insuffisamment } \\
\text { satisfait(e) }\end{array}$ & $\begin{array}{c}\text { Pas du } \\
\text { tout } \\
\text { satisfait(e) }\end{array}$ \\
\hline $\begin{array}{c}\text { Estimez-vous être capable de mettre en } \\
\text { application, à votre poste, les acquis de la } \\
\text { formation? }\end{array}$ & $80 \%$ & $20 \%$ & - & - \\
\hline $\begin{array}{c}\text { Le rythme de la formation vous a-t-il paru } \\
\text { satisfaisant ? }\end{array}$ & $90 \%$ & $10 \%$ & - & - \\
\hline $\begin{array}{c}\text { Les horaires étaient-ils adaptés au contenu de } \\
\text { la formation? }\end{array}$ & $90 \%$ & $10 \%$ & - & - \\
\hline $\begin{array}{c}\text { Le programme de la formation a-t-il été } \\
\text { respecté ? }\end{array}$ & $100 \%$ & $0 \%$ & - & - \\
\hline $\begin{array}{c}\text { L'animation a-t-elle facilité les échanges } \\
\text { entre participants ? }\end{array}$ & $100 \%$ & $0 \%$ & - & - \\
\hline $\begin{array}{c}\text { Les formateurs vous ont-t-ils paru } \\
\text { compétents ? }\end{array}$ & $100 \%$ & $0 \%$ & - & - \\
\hline $\begin{array}{c}\text { Les ressources pédagogiques (équipement, } \\
\text { supports, documentation...) ont-elles été } \\
\text { adéquates? }\end{array}$ & $100 \%$ & $0 \%$ & - & - \\
\hline $\begin{array}{c}\text { Vos attentes personnelles ont-elles été } \\
\text { satisfaites? }\end{array}$ & $90 \%$ & - & $10 \%$ & - \\
\hline
\end{tabular}

Source : Fiches d'évaluation de la formation des formateurs sur l'andragogie appliquée aux stratégies de mise en cuvre des ODD

Ainsi, $80 \%$ des participants estiment être capables de mettre en application, dans leur position professionnelle, les acquis de la formation. Le rythme de formation, les horaires de déroulement et le programme de la formation ont été en phase avec les attentes des apprenants dont le niveau de satisfaction, sur ces trois aspects, varie entre 90 et $\mathbf{1 0 0 \%}$. Enfin, l'animation andragogique, les profils des formateurs et les ressources pédagogiques fournies ont donné aussi une importante satisfaction aux apprenants. Il est ressorti un haut niveau de réalisation des attentes des apprenants $(\mathbf{9 0} \%)$ et une moyenne globale élevée, soit $\mathbf{( 9 3 , 7 5} \%$ ) de satisfaction des participants par rapport aux capacités en matière d'andragogie et de prise en compte des ODD dans les pratiques.

Les apprenants, tout en étant issus de divers domaines, se sont prononcés sur la formation; ce qu'il faut en retenir est dans l'encadré 3.

Encadré 3 : Les participants donnent leur avis sur le déroulement de la formation sur l'andragogie

La tenue de cette formation préalable à la mise en œuvre des sessions de formation continue nous a confortés en tant que experts-formateurs en 
Développement Durable, parce que la formation nous a donné une
importante opportunité de nous rendre compte de la pertinence d'utiliser les
outils et méthodes andragogiques pour dispenser une formation pour adulte.
Nous, participants à cette formation, sommes des acteurs de mise en œuvre
des changements qui doivent s'opérer dans la vie des citoyens au quotidien
pour que les bonnes pratiques qui doivent nous sauver et sauver notre
environnement s'installent. Donc, il est très important de maximiser les
chances d'atteinte des objectifs de la formation en misant sur des aspects
pratiques et concrets, comme cela a été le cas pendant ladite formation. Une
telle prise de conscience par les experts-formateurs que nous sommes nous
permettra de moduler au minimum les développements théoriques (dans le
déroulement des sessions de formation continue des cadres) avec les
méthodes utilisées en andragogie (questions-réponses, jeu de rôles,
brainstorming, études de cas pratiques, simulation, partage d'expériences,
etc.

En somme, sur l'ensemble des deux dimensions de la session de formation (andragogie appliquée et stratégies de mise en œuvre des ODD), les participants ont indiqué avoir eu satisfaction à un haut niveau. Quel est le point de vue des apprenants sur l'organisation matérielle de la formation?

\section{Niveau de satisfaction chez les participants par rapport à l'organisation matérielle de la formation}

L'organisation matérielle et technique de la formation intervient beaucoup dans l'atteinte des résultats escomptés. Ainsi, les aspects non directement liés aux pratiques andragogiques et des ODD ont été également abordés dans le questionnaire. Ces aspects comprennent les conditions d'organisation (la logistique), le cadre et l'environnement physique de la formation, etc.

Tableau 3. Niveau de satisfaction chez les participants par rapport à l'organisation matérielle de la session

\begin{tabular}{|c|c|c|c|c|}
\hline $\begin{array}{c}\text { Niveau de satisfaction par rapport aux } \\
\text { modalités et à l'organisation matérielle } \\
\text { de la session de formation }\end{array}$ & $\begin{array}{c}\text { Tout à } \\
\text { satisfait(e) }\end{array}$ & $\begin{array}{c}\text { En partie } \\
\text { satisfait(e) }\end{array}$ & $\begin{array}{c}\text { Insuffisamment } \\
\text { satisfait(e) }\end{array}$ & $\begin{array}{c}\text { Pas du tout } \\
\text { satisfait(e) }\end{array}$ \\
\hline Conditions d'organisation (logistique) & $95 \%$ & $5 \%$ & $\%$ & - \\
\hline Qualité générale de la salle & $95 \%$ & $5 \%$ & - & - \\
\hline $\begin{array}{c}\text { Confort (thermique, acoustique, etc.) des } \\
\text { participants }\end{array}$ & $98 \%$ & $2 \%$ & - & - \\
\hline $\begin{array}{c}\text { Qualité des pauses-cafés et des repas } \\
\text { servis par le service traiteur }\end{array}$ & $90 \%$ & $10 \%$ & - & - \\
\hline
\end{tabular}

Source : Fiches d'évaluation de la formation des formateurs sur l'andragogie appliquée aux stratégies de mise en ruvre des $O D D$

Au niveau du tableau 3, il est constaté également un niveau de satisfaction élevé (entre 90 et 98\%) par rapport aux quatre questions posées. Il 
est utile de relever ici que le niveau de satisfaction pour ce groupe de variables est plus élevé $(94,5 \%)$ que pour les groupes de variables précédents. Cependant, il y a la qualité des pauses-cafés et des repas servis par le service traiteur qui n'a pas suffisamment comblé les attentes de $10 \%$ des participants.

\section{Discussion}

Les résultats dévoilent que $80 \%$ des formateurs enquêtés pensent être, désormais, capables de mieux enseigner aux apprenants adultes. Ces formateurs assurent, à cet effet, que le rythme de formation, les horaires de déroulement et le programme de la formation, leur ont permis d'atteindre les objectifs de la formation. Il faut plutôt asseoir un dispositif éducatif et de formation qui tienne compte des besoins particuliers des adultes plus difficiles à satisfaire, promeut Schwartz (1968).

L'animation andragogique, les profils des formateurs et les ressources pédagogiques mises à disposition ont favorisé un climat d'apprentissage sain. Pour arriver à ce résultat au niveau de l'apprenant, précise Schwartz (1968), il importe de ne pas dissocier la formation de l'adulte de « sa vie affective réelle qui comprend à la fois des réalités professionnelles, familiales, sociales, culturelles » (p.43). En fait, la définition d'objectifs appropriés est la clé de la réussite d'une formation. Tout le processus de gestion de la formation répond aux directives qu'impliquent les objectifs d'apprentissage clairement définis, qui ne peuvent que correspondre aux attentes des participants, puisque, découlant de leurs besoins. Le contenu de la formation se conçoit en modules qui sont chacun liés à un objectif pré défini. Il est soigneusement élaboré pour capter les apprenants. Il faut, pour stimuler les facultés des participants, déployer des méthodes/techniques andragogiques telles que le jeu de rôles, la simulation, la démonstration, les études de cas... en passant par les stades de travail individuel, de travail en groupe, puis vient la validation des connaissances coconstruites en plénière. Toutes ces considérations font remarquer que l'andragogie englobe l'éducation en milieu socio-professionnel et l'éducation post-universitaire de Schwartz (1968). Parallèlement, en reprenant les idées de Schmidt (2018), il apparaît que l'andragogie permet le changement de comportements de manière consentante indispensable au développement de nouvelles aptitudes et attitudes, nécessaires à la mise en œuvre des ODD.

Par ailleurs, les conditions d'organisation, le confort thermique, acoustique, etc. dans la salle de formation, les repas et des collations ont permis aux participants d'avoir de l'entrain à suivre les modules proposés. Plus de $93,75 \%$ sont satisfaits de tout cet ensemble. Il ne faut pas oublier qu'il convient d'entretenir la motivation et la détermination de l'adulte à apprendre, pour peut-être aller plus loin que Health Nexus santé (2003) ou Knowles (1970-1990) cité par Meilleur (2018), ceci engage aussi l'environnement 
physique d'apprentissage. Hutchinson (2003) cité par Nelson (2020) trouve qu'il est indispensable de considérer « les paramètres suivants : luminosité et température de la salle de formation, confort des sièges, bruit de fond dans l'environnement d'apprentissage, distractions visuelles, tutoriels, séminaires, conférences ».

Schmidt (2018) rappelle combien apprendre à un adulte est différent de comment ça se passe avec des enfants et adolescents en ce sens que l'adulte est de nature rigide et donc pas vraiment ouvert aux changements. Il semble plus qu'opportun de n'omettre aucun facteur pouvant lui permettre d'être à son aise pour travailler et acquérir les connaissances et attitudes qui sont attendus de lui. L'apprenant est vraiment au cœur du processus de formation.

L'enquête auprès des participants de l'atelier tenu dans ce centre de formation des formateurs sur les ODD, a démontré que les participants, tous des hauts cadres de l'administration publique, ont éprouvé un haut niveau de satisfaction, principalement par rapport aux attentes et aux acquisitions. Ainsi, nous pensons que par l'approche andragogique (en termes de flexibilité, de stratégies de motivation et d'outils didactiques, etc.) adoptée, l'atelier a atteint les objectifs de la formation. L'étude confirme, alors, que sur les quatre niveaux du modèle proposé par Kirkpatrick (1979) cité par Yennek (2015), au moins le premier niveau « satisfaction » a été atteint au cours de la formation. Comme le propose ce modèle, les résultats positifs obtenus avec le remplissage des formulaires d'évaluation à la fin de l'atelier et les perceptions des apprenants laissent supposer que la formation a favorisé l'apprentissage. Le respect des principes andragogiques a sans doute contribué aux réactions positives des participants.

Selon Yennek (2014) cité par Yennek (2015), la satisfaction en formation est considérée comme un indicateur d'efficience de la formation donc de la qualité du processus sous-jacent à l'atteinte des résultats escomptés. Elle doit, selon Yennek (2015), tenir compte des aspects les plus déterminants pour «prédire l'apprentissage » (au sens du niveau 2 de Kirkpatrick, 1979); l'un de ces aspects peut être l'utilité perçue, pour " prédire le transfert » (au sens du niveau 3 de Kirkpatrick, 1979). La figure ci-dessous comprend les grands points à développer dans une évaluation de formation :

Figure. Modèle de l'évaluation de la formation de Kirkpatrick

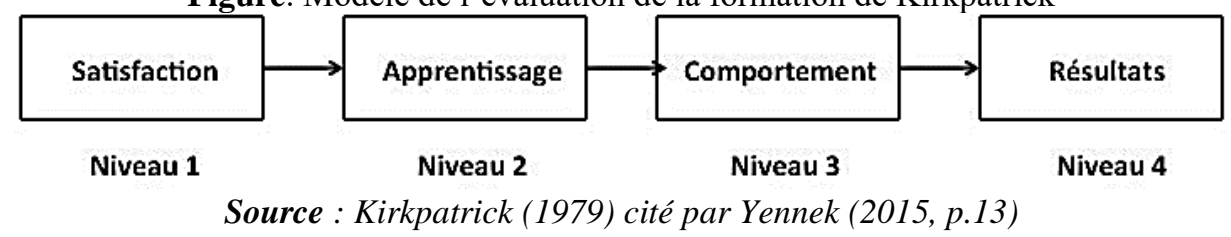

- Niveau 1: satisfaction des participants sur le déroulement de la formation ; 
- Niveau 2 : apprentissages de la formation en termes d'acquisition des connaissances, compétences ou de changement d'attitudes ;

- Niveau 3 : comportement en termes de changements observés dans le milieu professionnel ;

- Niveau 4: résultats où les indicateurs comme l'augmentation de la productivité, l'amélioration de la qualité du travail sont mesurées pour percevoir l'impact réel de la formation sur l'organisation.

Goldstein et Ford (2002, p. 153) cités par Yennek (2015) ont mentionné que " même s'il n'y a pas de relation consistante entre la satisfaction des apprenants et la performance (au sens du niveau 2 de Kirkpatrick, 1979), peu de gens soutiendraient l'idée de lancer un programme de formation qui aboutirait à l'insatisfaction des participants ».

\section{Conclusion}

Le présent article décrit la pratique éducative dans la formation en andragogie des formateurs leur permettant de poursuivre avec des modules propres à un champ donné, ici, les stratégies de mise en œuvre des ODD. Un point a été fait des lignes abordées au cours de cette formation, de ce qu'en général, les apprenants formateurs ont retenu. Le haut niveau de satisfaction obtenu avec ce dispositif de formation témoigne de ce que les principes andragogiques ont été largement respectés. Il faut rappeler que chaque phase du cycle de formation reconstitue les principes fondamentaux de l'andragogie. Il est certain que la formation doit envisager les activités en se référant à l'environnement professionnel duquel proviennent les participants apprenants.

Cette recherche fournit une capitalisation des stratégies et initiatives pour motiver et renforcer les capacités des formateurs, ici, des cadres supérieurs des administrations publiques et privées du Bénin. Ces formateurs assureront mieux leur rôle auprès des acteurs publics ou sociaux en intégrant les ODD aux projets, programmes, stratégies et politiques administratives et de développement. En outre, l'approche andragogique recommande de mesurer les apprentissages avant, pendant et après la formation, ainsi qu'à moyen terme (trois à six mois après la formation). Ce sont les résultats de l'évaluation du niveau de satisfaction des participants à la fin de la session de formation des formateurs qui font l'objet de connaissance et de compétence dans cet article. L'approche andragogique pour former les formateurs et renforcer leurs capacités dans la compréhension et l'interprétation des ODD est bien adaptée comme stratégie d'atteinte des objectifs de formation des formateurs. 


\section{Remerciements}

Les remerciements vont à l'endroit de l'Ecole normale d'Administration (ENA) du Bénin qui a abrité tous les travaux de l'atelier; à l'Institut de la Francophonie pour le Développement durable (IFDD) et à l'Organisation internationale de la Francophonie (OIF), pour leur accompagnement technique et financier; à tous les formateurs des formateurs pour leur collaboration; et à tous les participants formateurs pour leur assiduité à la formation et leur intérêt pour la phase d'évaluation; à tous les autres acteurs de l'atelier des formateurs pour la mise en œuvre des Objectifs de Développement durable qui s'est déroulé en juillet 2021. Tous ceux-là ont participé à la réalisation de l'atelier qui ont permis l'existence de cet article.

\section{References:}

1. Akouété-Hounsinou, F. (2021). Typologie et qualité pédagogique des dispositifs hybrides de formation dans les universités du Bénin. ReSciLaC, $\mathrm{N}^{\circ} 13$, vol. 2, p.153-169.

2. Cieuta, P. (2019). Formation de formateurs, fiche 11 : Andragogie. https://www.cahiers-pedagogiques.com/wpcontent/uploads/2019/01/organiser_la_cooperation_entre_eleves__fiche_11_andragogie.pdf

3. Expilly, F. (2017). Adopter une posture de formateur : rôle, attitudes, le groupe, la relation d'aide. [Vidéo]. https://www.youtube.com/watch?v=i3gZMQRvh9g

4. Health Nexus santé. (2003). Mieux comprendre la formation aux adultes, l'andragogie : une science imprégnée d'humanisme, Le BlocNotes. https://www.leblocnotes.ca/node/251

5. Likert, R. (1961). New Patterns of Management. New-York : McGraw-Hill Book company, ix, 279 pp.

6. Meilleur, C. (2018). L'adulte : un apprenant distinct. https://knowledgeone.ca/the-adult-a-distinct-learner/?lang=fr

7. Nelson, S. (2020). Environnement physique d'apprentissage et pratiques pédagogiques. Le cas des écoles de la Grand'Anse. Dans L. Rochambeau (dir.), Le handicap à l'école haïtienne. Résultats préliminaires d'une recherche-action dans le grand Sud d'Haïti à la suite de l'ouragan Matthew (pp. 71-81). Québec : Éditions science et bien commun.

https://scienceetbiencommun.pressbooks.pub/handicaphaiti/chapter/e nvironnement-physique-dapprentissage-et-pratiques-pedagogiques/

8. Schmidt, J.-P. (2018). Apprendre tout au long de la vie ? Des possibilités pour tout adulte. Analyse UFAPEC, $\mathrm{n}^{\circ} 08.18$. https://www.ufapec.be/files/files/analyses/2018/0818-Andragogie.pdf 
9. Schwartz, B. (1968). Réflexions sur le développement de l'éducation permanente. Revue française de pédagogie, volume 4, 1968. pp. 3244. https://www.persee.fr/doc/rfp_0556-7807_1968_num_4_1_1762

10. Yennek, N. (2015). La satisfaction en formation d'adultes. In Savoirs, (38), pp.9-54. Consulté le 10 novembre 2021 à l'URL https://www.cairn.info/revue-savoirs-2015-2-page-9.htm

11. Zaoré, R. (2007). Les principes andragogiques, le rôle de formateur. Programme de formation en hygiène et salubrité. https://www.msss.gouv.qc.ca/professionnels/documents/hygiene-etsalubrite/1_1_Caracteristiques_apprenant_adulte.doc 